\title{
Endoscope-assisted hemispherotomy: translation of technique from cadaveric anatomical feasibility study to clinical implementation
}

\author{
Kathryn Wagner, MD, ${ }^{1,2}$ Francisco Vaz-Guimaraes, MD, ${ }^{1}$ Kevin Camstra, MS, ${ }^{1}$ and \\ Sandi Lam, MD, MBA ${ }^{1,2}$ \\ 'Department of Neurosurgery, Baylor College of Medicine; and 2Division of Pediatric Neurosurgery, Texas Children's Hospital, \\ Houston, Texas
}

\begin{abstract}
OBJECTIVE Appropriately chosen candidates with medically refractory epilepsy may benefit from hemispheric disconnection. Traditionally, this involves a large surgical exposure with significant associated morbidity. Minimally invasive approaches using endoscopic assistance have been described by only a few centers. Here, the authors report on the feasibility of endoscope-assisted functional hemispherotomy in a cadaver model and its first translation into clinical practice in appropriately selected patients.
\end{abstract}

METHODS Three silicone-injected, formalin-fixed cadaver heads were used to establish the steps of the procedure in the laboratory. The steps of disconnection were performed using standard surgical instruments and a straight endoscope. The technique was then applied in two patients who had been referred for hemispherectomy and had favorable anatomy for an endoscope-assisted approach.

RESULTS All disconnections were performed in the cadaver model via a $4 \times 2$-cm paramedian keyhole craniotomy using endoscopic assistance. An additional temporal burr hole approach was marked in case the authors were unable to completely visualize the frontobasal and insular cuts from the paramedian vertical view. Their protocol was subsequently used successfully in two pediatric patients. Full disconnection was verified with postoperative tractography.

CONCLUSIONS Full hemispheric disconnection can be accomplished with minimally invasive endoscope-assisted functional hemispherotomy. The procedure is technically feasible and can be safely applied in patients with favorable anatomy and pathology; it may lead to less surgical morbidity and faster recovery.

https://thejns.org/doi/abs/10.3171/2018.8.PEDS18349

KEYWORDS hemispherotomy; hemispherectomy; epilepsy; seizure surgery; endoscopic; endoscope-assisted; keyhole

$\mathrm{H}$ EMISPHERIC disconnection is an effective surgical option for carefully selected patients with disabling, medically refractory epilepsy. Ideal candidates are typically those with an etiology attributable to diffuse abnormalities of one hemisphere. This can include perinatal strokes, developmental or migration disorders, syndromes such as Sturge-Weber, or progressive processes such as Rasmussen's encephalitis. ${ }^{8,21,30}$ Seizure freedom rates can be as high as $90 \%$ in the long term, depending on the pathology treated and the technique of hemisphere disconnection. ${ }^{3,8,10,14,16,29}$

A progression of disconnection techniques has been described over the past century, each addressing efforts to minimize exposure and complications while still accessing target deep structures. Anatomical hemispher- ectomy involved full exposure and removal of an entire hemisphere ${ }^{6,12,17}$ but yielded an unacceptable complication profile including superficial cerebral hemosiderosis and hydrocephalus. ${ }^{9,20,23}$ Functional hemispherectomy reduced these complications by leaving some of the brain tissue in situ, ${ }^{22,32}$ the recognition that disconnection but not resection could produce results similar to those obtained with anatomical hemispherectomy provided the basis for Villemure's periinsular hemisphereotomy ${ }^{31}$ and Delalande's vertical hemisphereotomy. Many modifications have subsequently been described, all with the goal of minimizing both excision and exposure while maximizing disconnection. ${ }^{24-26}$ Regardless of the technique used, full hemispherotomy entails disconnection of the corpus callosum, frontobasal white matter, insular white matter, 


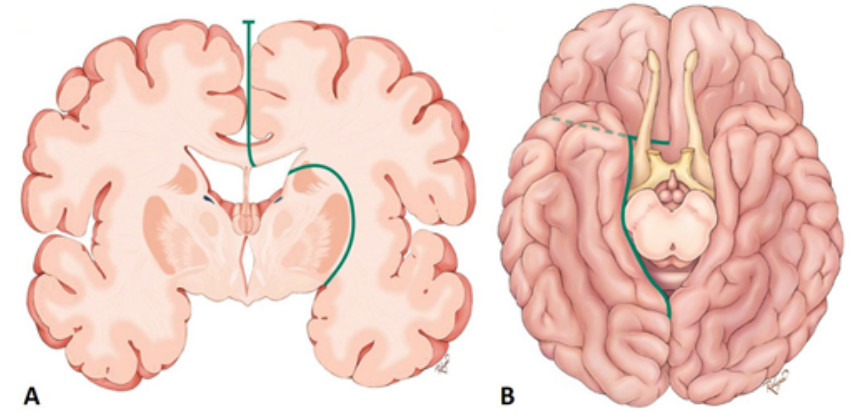

FIG. 1. Disconnections for functional hemispherotomy. A: Callosotomy and insular disconnection. B: Frontobasal and mesial temporal lobe disconnections. Green lines indicate lines of disconnection. Image created by Katherine Relyea, MS, CMI, and printed with permission from Baylor College of Medicine. Figure is available in color online only.

and medial temporal lobe structures, including the hippocampus and fornix (Fig. 1). These approaches require a potentially large craniotomy for an adequate operative corridor and good visualization of the anatomy. This extensive surgical exposure can lead to increased surgical morbidity, including hypothermia, coagulopathy and blood loss necessitating transfusion, pain and swelling at the surgical site, complicated wound infections/revisions, and prolonged patient recovery. ${ }^{15}$

In order to minimize the surgical exposure and decrease perioperative morbidity, several groups have reported the development of endoscope-assisted disconnection procedures ranging from corpus callosotomy to complete hemispherectomy (Fig. 2).,4,5,19,27 The evolution of surgical instruments and techniques has made cranial surgery possible through smaller operative corridors. We aimed to investigate the anatomical feasibility of an endoscope-assisted surgical technique for hemispherotomy at our institution by beginning with a cadaveric study. We then transitioned our technique into clinical practice, and here we present two patients in whom hemispherotomy with complete disconnection was performed via our minimally invasive endoscope-assisted approach.

\section{Methods \\ Cadaver Study}

Three silicone-injected cadaveric heads were utilized in this study, which was conducted in our neurosurgical anatomy laboratory. Standard surgical instruments, microsurgical instruments, and endoscopic instruments were available for use in the anatomy lab. A Karl Storz (Karl Storz $\mathrm{GmbH}$ ) $0^{\circ}$ straight endoscope was selected for use, with high-definition images from the endoscope camera viewed on a screen. Video recordings and still photographs were captured.

We predefined the steps of the operation with disconnections including corpus callosotomy, frontobasal disconnection, insular cut (and temporal horn unroofing), and hippocampal (fornix) disconnection (Table 1). Thus, the major anatomical points of interest included the corpus callosum, insula, temporal horn, hippocampus, and tentorial edge; the frontobasal disconnection would require vi-

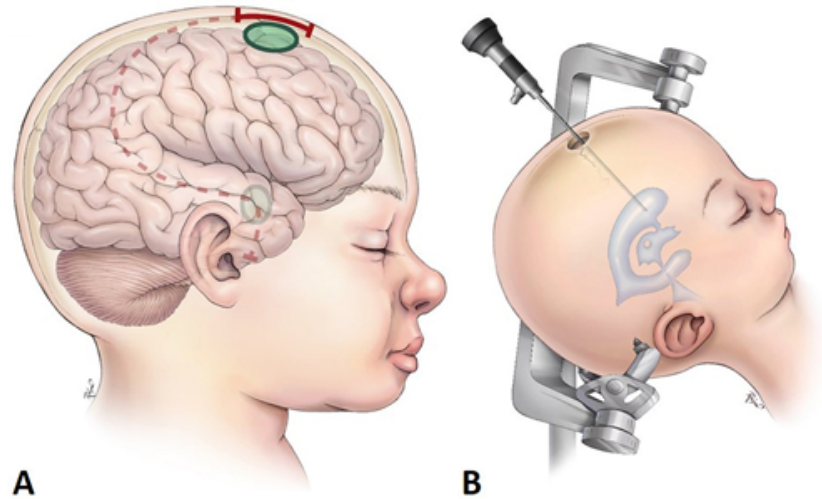

FIG. 2. A: Incision and mini craniotomy (solid red line) compared to the standard incision for open hemispherotomy (dashed red line). Green circle indicates burr hole. Ghosted light green burr hole shows a supplemental access point to the temporal horn through the middle temporal gyrus. B: Supine, fixed patient position for endoscope-assisted procedure. Image created by Katherine Relyea, MS, CMI, and printed with permission from Baylor College of Medicine. Figure is available in color online only.

sualization of the ipsilateral anterior cerebral arteries, middle cerebral arteries, and internal carotid artery. Surgical planning was based on anatomical landmarks and previously published measurements. ${ }^{1,5,27,28}$ Disconnections were verified using the endoscope through a temporal burr hole.

\section{Clinical Application}

After successful hemispherotomy disconnection in three cadaveric heads in the neuroanatomy laboratory, the technique was applied in two pediatric patients with intractable epilepsy who were candidates for open hemispherectomy. Selected patients had had perinatal strokes leading to cystic spaces in the pathological hemisphere, thereby allowing a larger working corridor. The surgical technique is described, as are patient histories, images, and outcomes.

Cadaver lab study did not require IRB approval at our institution. Moreover, neither the use of endoscopic tools nor the anatomical goals of the hemispherotomy surgery were new or experimental. The way we approached, planned, and executed the procedure was technically novel to us. As surgical approach and technique often evolve over time, our endeavor was considered a technical shift and refinement rather than the discovery of a new treatment. Because we were not using a new tool, applying a new technology, or performing an entirely new procedure based on novel pathophysiological underpinnings, IRB approval was not required. Retrospective chart review of the clinical cases for this study did receive IRB approval.

\section{Results \\ Cadaver Study}

Mayfield pins and head holder were used for fixation and positioning of the cadaveric heads. The positioning was supine with slight flexion. Palpation and anatomical landmarks were used to locate the coronal suture, and a straight $5-\mathrm{cm}$ incision was marked extending $3 \mathrm{~cm}$ ante- 
TABLE 1. Disconnections required for hemispherotomy

\begin{tabular}{c}
\hline Hemispherotomy Disconnections \\
\hline Corpus callosum (rostrum to splenium) \\
\hline Frontobasal fibers (uncinate, occipitofrontal fasciculi) \\
\hline Corona radiata (projection fibers, internal capsule) \\
\hline Mesial temporal lobe (hippocampus, fornix) \\
\hline
\end{tabular}

rior and $2 \mathrm{~cm}$ posterior to the coronal suture (Fig. 3A). A standard question mark-shaped incision for a traditional frontoparietotemporal craniotomy was provisionally marked, with the inferior vertical limb anterior to the ipsilateral ear down to the level of the zygoma (Fig. 3C). This temporal incision would allow a small craniotomy for access to the temporal horn of the lateral ventricle if needed, as well as provide a corridor for visual confirmation of the frontobasal disconnection. A $4 \times 2-\mathrm{cm}$ keyhole craniotomy was made with a high-speed drill, with the medial border exposing the midline (Fig. 3E). The dura mater was opened, and the flap was retracted toward the sagittal sinus.

\section{Corpus Callosotomy}

We performed an interhemispheric approach to the corpus callosum with endoscopic visualization and dynamic retraction using a Penfield instrument (Fig. 4A). The pericallosal arteries were identified overlying the corpus callosum, and the midline surgical corridor was defined between the vessels. The corpus callosotomy was done with controllable suction, microsurgical instruments, and bipolar cautery. The lateral ventricle was entered, with visual identification of the ipsilateral intraventricular anatomy landmarks, including the septal vein, thalamostriate vein, choroid plexus, and foramen of Monro.

We completed the callosotomy with the anterior extent exposing the anterior cerebral arteries via subpial resection of the white matter of the genu (Fig. 4B) and the posterior extent visualizing the pineal cistern and internal cerebral veins via subpial resection of the white matter of the splenium (Fig. 4C). The anatomy was easily identifiable during this portion of the study, as in an open callosotomy or a transcallosal approach to ventricular pathology.

\section{Disconnection of Insular White Matter}

The view from inside the ventricle requires identification of intraventricular landmarks, including the foramen of Monro, caudate nucleus, choroid plexus, atrium, and temporal horn. The temporal horn was accessed from the atrium of the lateral ventricle (Fig. 4D). We followed the choroid plexus and ependymal walls and advanced a rectangular cottonoid patty from the atrium to the ipsilateral temporal horn.

We used bipolar cautery, microscissors, microinstruments, and controllable suction to unroof the temporal horn of the lateral ventricle and disconnect the cortical projection fibers, beginning along the ventricular margin of the caudate. This anatomically corresponds with disconnection of the internal capsule and proceeds from inside the ventricle outward. As the disconnection pro-
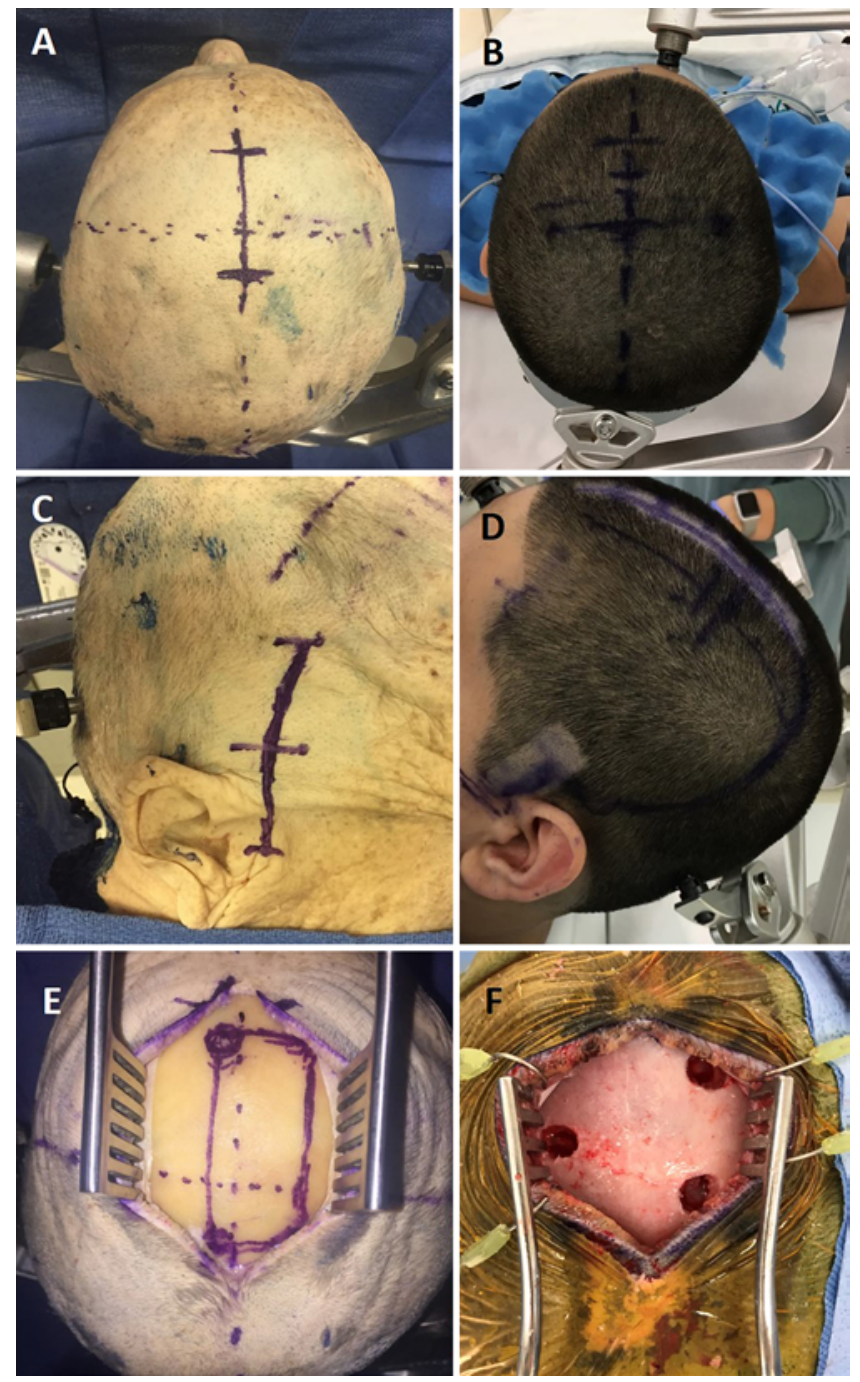

FIG. 3. Positioning and opening in the cadaver head (A) and the patient (B, for left hemispherotomy). Midline and coronal suture marked in the cadaver head (C) for right hemispherotomy and middle temporal gyrus access. Planned incisions (D) for case 1 for right hemispherotomy by external landmarks and neuronavigation. Note the possible temporal incision and full craniotomy incision indicated with marking pen. Midline coronal suture and right craniotomy marked on the cadaver head $(E)$. For left hemispherotomy $(\mathbf{F})$ : two burr holes over the sagittal sinus with one lateral burr hole on the coronal suture for a 4-cm-long $\times 2-\mathrm{cm}$-wide craniotomy in case 1 . Figure is available in color online only.

gressed, especially in specimens without significant ventriculomegaly (and thus a smaller working area), it was imperative to continually identify known landmarks (i.e., falx, choroid plexus, ependyma) to maintain orientation. The aforementioned cottonoid patty served as an additional point of reference in the temporal horn of the lateral ventricle. In the anatomy lab, neuronavigation was not available, though stereotactic navigation would be an additional intraoperative instrument.

\section{Hippocampectomy}

After visualizing the entire lateral ventricle, including the temporal horn, and completing the insular disconnec- 

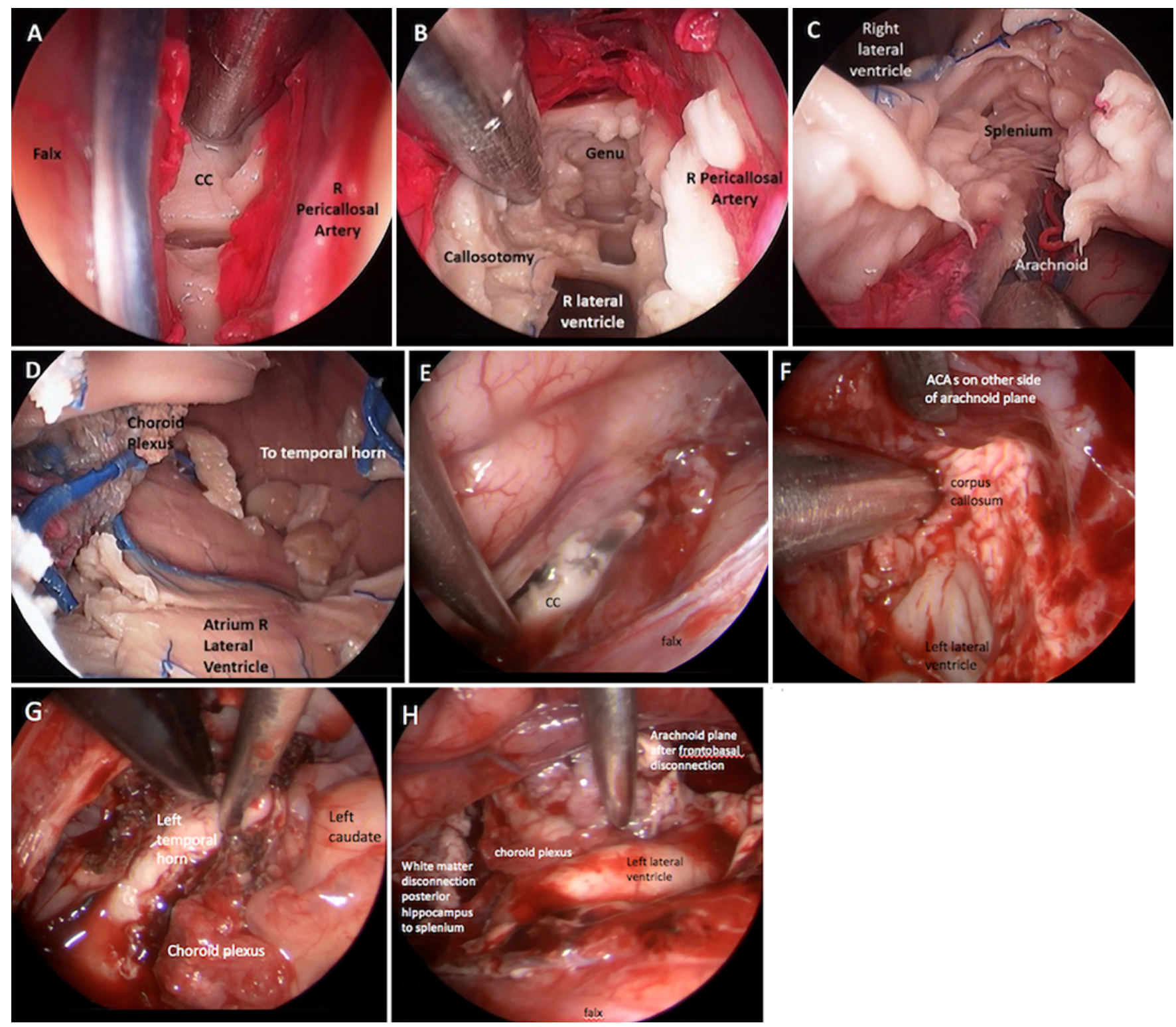

FIG. 4. Endoscopic disconnection of the right hemisphere in a cadaver model (A-D) and intraoperative endoscopic pictures (E-H). A: Parafalcine approach to the corpus callosum. B: Callosotomy at the genu. C: Callosotomy at the splenium with a view of the pineal cistern deep to the arachnoid membrane. D: Atrium of the right lateral ventricle, with the choroid plexus leading into the temporal horn. E: Interhemispheric approach to the corpus callosum. F: Callosotomy at the genu. G: Exposure into the left temporal horn. $\mathrm{H}$ : View into the left lateral ventricle with white matter disconnections and associated arachnoid planes. ACA = anterior cerebral artery; $\mathrm{CC}=$ corpus callosum; $\mathrm{R}=$ right. Figure is available in color online only.

tion, the hippocampus was identified. The white matter of the hippocampus was disconnected via subpial resection, visualizing the posterior cerebral artery and the third cranial nerve past the tentorial edge. The white matter disconnection proceeded posteriorly in the subpial plane to the tail of the hippocampus, continued to the posterior fornix, and extended until it met the area where the splenium had been resected.

\section{Frontobasal Disconnection}

The frontobasal disconnection and insula cuts were made anterior and lateral to the caudate. Subpial resection was carried along the ipsilateral anterior cerebral artery to the bifurcation of the internal carotid artery. Anteriorly, this was connected to the previously unroofed temporal horn.

As the procedure progressed, the disconnected hemispheric cortex required some dynamic retraction by the surgical assistant to allow passage of the working surgical instruments. We frequently referred to known normal anatomical structures such as the major arteries, intraventricular ependymal surface, falx, tentorial edge, and choroid plexus to ensure the maintenance of surgical orientation. At this point, a complete hemispherotomy had been per- 


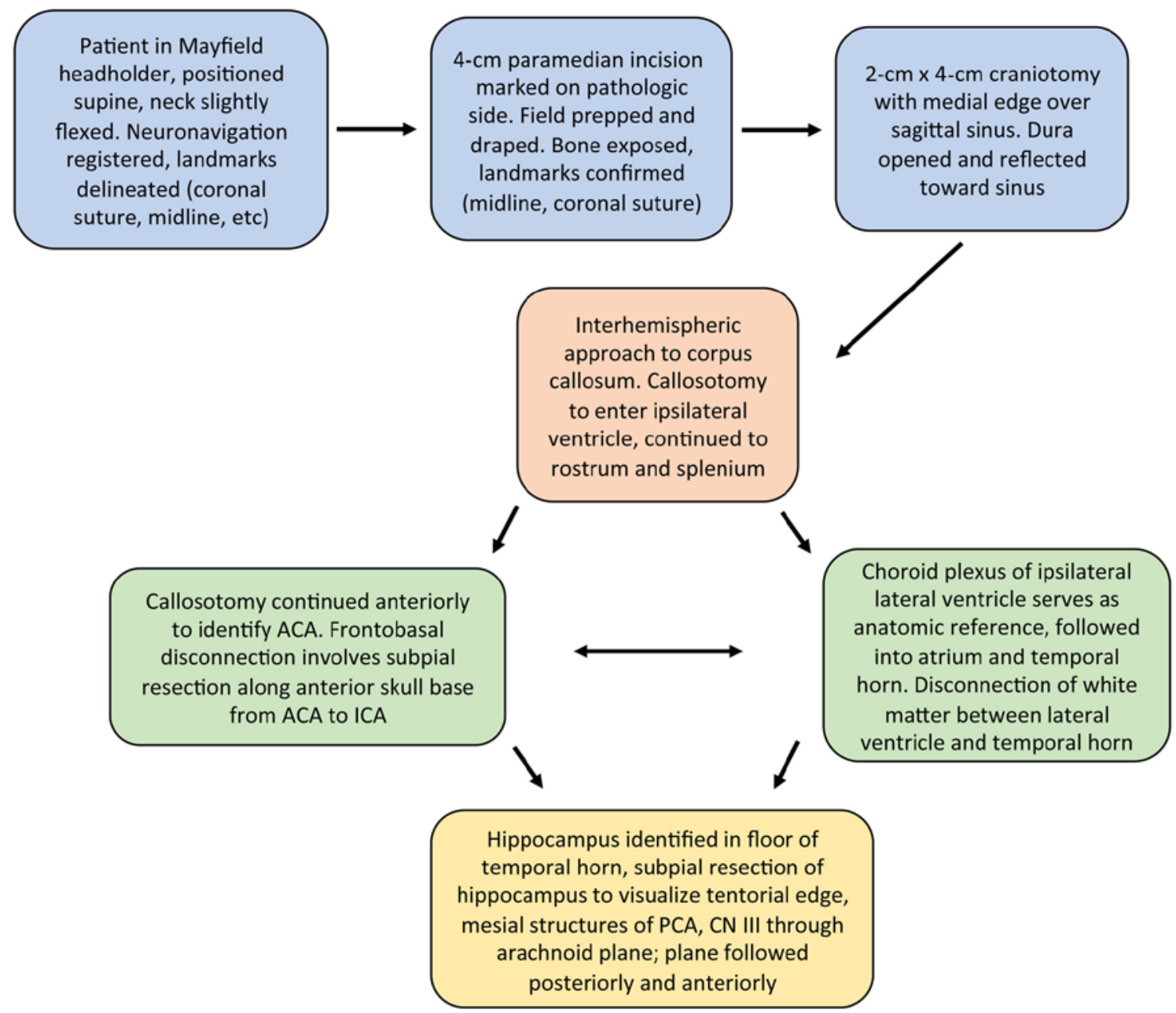

FIG. 5. Workflow of endoscope-assisted hemispherotomy. Patient is positioned and the operative field is exposed. Callosotomy is performed to enter the ventricle. The frontobasal disconnection and temporal horn unroofing can proceed in a dynamic order. Finally, a partial hippocampectomy for mesial temporal disconnection is performed. Figure is available in color online only.

formed, with endoscopic visualization of all disconnections.

\section{Temporal Horn Access}

As a possible alternative for access to the temporal horn and hippocampus in the event that full hippocampectomy and frontobasal disconnection could not be completed, we tested a middle temporal gyrus approach. The vertical limb of the traditional question mark-shaped skin incision was opened, and a $2 \times 2-\mathrm{cm}$ burr hole superior to the zygoma was created with a high-speed drill. The dura was opened in a cruciate fashion. A corticectomy was performed in the middle temporal gyrus, parallel to the middle fossa floor, until the temporal horn was entered. The opening into the ventricle was extended until it was possible to inspect the hippocampal resection bed and to visualize full resection from the head to the tail. The tentorial edge, posterior cerebral artery, and third cranial nerve were visualized through the arachnoid from this approach via endoscopic visualization. Posteriorly, we visualized the choroid plexus in the atrium of the ipsilateral lateral ventricle. Access and visualization anteriorly to the internal cerebral artery was also ergonomic and feasible through this approach should it be needed for disconnection.

Thus, a complete hemispherotomy disconnection was achieved. The workflow and progression of disconnections are shown in Fig. 5. We recognized that a solo surgeon was not able to easily perform the disconnections with instruments through the endoscopic working channel, nor to have safe control of blood vessels for hemostasis. Thus, we developed the endoscope-assisted method, a three-hand technique in which the surgical assistant holds the endoscope, leaving the surgeon free to operate with both hands. With the endoscope-assisted method, all steps of the surgery could be achieved in the cadaveric study. We confirmed the anatomy and disconnections with endoscopic visualization. Finally, we finished by performing a standard craniotomy on the cadaver models to directly verify anatomy and disconnections.

\section{Clinical Application}

The endoscope-assisted technique was translated into 
clinical practice in two patients who had been referred for hemispherotomy surgery. We recognized the steep learning curve when converting a large open craniotomy surgery to a minimal access surgery, and this factored into patient selection. Given favorable anatomy with a generous ventricular size and relative paucity of cortical tissue, our patients were appropriate candidates for application of the endoscope-assisted hemispherotomy procedure.

In practice, a multidisciplinary epilepsy conference is convened weekly for surgical epilepsy candidates to review their history, physical examination, imaging, epilepsy workup, and treatment options. Three pediatric epilepsy neurosurgeons attend this conference. A spectrum of surgical options for any proposed procedure is reviewed, taking into account known evidence, specific anatomy, and case details, as well as surgeon and family preferences. The possibility of a smaller cranial opening for hemispherotomy was introduced first in detail to the team and then incorporated into the discussion.

Rationale for surgery and surgical options were reviewed by the epileptologist along with the family. The same information together with additional surgical details and the expected perioperative timeline was discussed at the neurosurgery appointment. The overall goal of surgery was to safely achieve the disconnective cuts for hemispherotomy. The option of a smaller opening with the use of an endoscope was discussed with the family. We were open and direct about the relative novelty of the application of this surgical technique involving a smaller craniotomy opening and use of the endoscope. We gave the parents the information, with the traditional standard frontoparietotemporal craniotomy represented as the standard surgery. The rationale for the endoscope-assisted hemispherotomy, pros and cons, and the contingency plans for converting to open craniotomy were reviewed. The families knew that their cases were the first and second to be undergoing this specific technique modification at our institution, and they shared the decision-making by opting into this modified surgical technique.

Discussion of each case was then held at the pediatric neurosurgery indications conference with the faculty, fellow, resident, and perioperative group to confirm surgical candidacy and evaluate the proposed surgical approach. Perioperative care was coordinated during the high-risk case conference with anesthesia staff and operating room nursing staff.

\section{Translation to the Operating Room}

After general anesthesia was induced, patients were positioned supine with the head fixed in a Mayfield head holder, with pin sites selected to allow for the paramedian incision over the coronal suture, with the option for conversion to an open question mark-shaped incision for frontotemporoparietal craniotomy. BrainLab (BrainLab AG) neuronavigation registration was undertaken. We verified that external landmarks corroborated with the BrainLab registration. We marked the midline and coronal suture for external reference. Using the BrainLab in-line trajectory view, we verified that the intended craniotomy opening could reach the genu, splenium, and ipsilateral temporal horn.
A paramedian 5-cm incision was marked $2 \mathrm{~cm}$ off the midline. We modified the incision to a paramedian location, as this facilitated the craniotomy. This line was typically over the coronal suture with approximately $3 \mathrm{~cm}$ anterior and $2 \mathrm{~cm}$ posterior to the coronal suture (Fig. 3B). A full question mark-shaped standard craniotomy for hemispherotomy incision was also marked, with the vertical line of the question mark falling within $1 \mathrm{~cm}$ of the ear, ending anterior to the tragus at the zygoma (Fig. 3D). This traditional open-incision marking would be used if the surgery needed to be extended to an open craniotomy. The vertical limb of the question mark shape drawn above the zygoma would serve as the area of access should the second burr hole for accessing the temporal horn become necessary. This entire standard question mark-shaped incision was prepped and draped into the sterile field.

The procedure was performed as previously described, without adverse events. After the scalp was opened and cranial sutures on the skull surface were visible, a $4-\mathrm{cm}-$ long by $2-\mathrm{cm}$-wide craniotomy was undertaken, with burr holes made on the coronal suture and sagittal suture (Fig. $3 \mathrm{~F})$ to expose the dura overlying the sagittal sinus and carefully free up the dura underneath the bone. Neuronavigation was used, with the surgical team being cognizant that distortion after access into the ventricle could lead to a decrease in accuracy. Intraoperative ultrasound was available in the event that additional verification of anatomy was required beyond direct endoscopic visualization and could be used to identify the ventricular anatomy, tentorial edge, and skull base. Though the equipment was on and ready, the use of intraoperative ultrasound was not required in our two clinical cases. Moreover, the temporal burr hole access was not needed; thus, the objectives of surgery were achieved entirely from the single paramedian exposure.

The cortical brain parenchyma in vivo was quite mobile after performing the callosotomy and entering the ventricle because of the large ventricular anatomy and thin cortical ribbons. Thus, the cortex could be gently retracted away from the operative field with wet cotton balls placed between the pathological hemisphere and the falx. The view through the endoscope was much more likely to become blocked by tissue in vivo than in the cadaveric anatomy lab. Therefore, we added controllable irrigation (ClearVision, Karl Storz GmbH), which allowed on-demand clearing of the endoscopic view. In order to maximize the limited working space, an angled endoscope camera (P3, Karl Storz $\mathrm{GmbH}$ ) was used. This ergonomic addition kept the assistant's hands away from the working field and corridors of the operative surgeon while still allowing manipulation and dynamic positioning of the endoscope.

After the procedure, a ventriculostomy catheter was left in place in the ipsilateral lateral ventricle via the interhemispheric exposure, exiting from an existing burr hole and tunneled away from the incision. Blood transfusions were not required. Both patients were extubated and observed in the pediatric intensive care unit setting overnight and transferred to a surgical unit on postoperative day 1 to begin mobilization and therapy evaluations. Both recovered well; they were discharged to home within 1 week without the need for CSF shunting and without demon- 

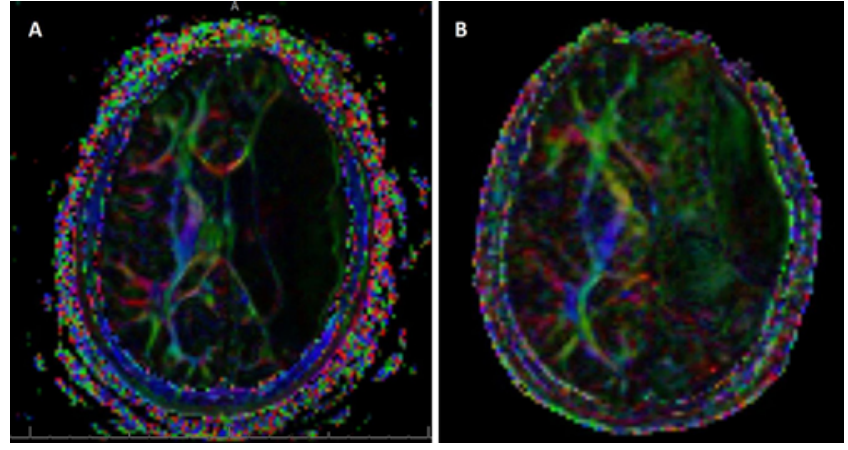

FIG. 6. Case 1. Preoperative baseline (A) and postoperative (B) tractography images showing full hemispheric disconnection. Figure is available in color online only.

strating a need for inpatient rehabilitation. Neither patient experienced postoperative fever. Postoperative MRI with diffusion tensor imaging showed complete disconnection of the fiber tracts (Fig. 6). At the 6-month follow-up, both patients had remained seizure free since surgery.

\section{Illustrative Cases \\ Case 1}

An 11-year-old boy had a history of cerebral palsy and intractable epilepsy secondary to perinatal stroke since the age of 8 months. Despite medical optimization, he was having up to five seizures per day. Comprehensive multidisciplinary epilepsy workup concluded that he had seizures originating from the left frontoparietal and posterior temporooccipital region propagating to the anterior temporal lobe. Magnetic resonance imaging showed structural abnormality of the left hemisphere with extensive cystic encephalomalacia consistent with the clinical history of infarction (Fig. 7A and B). He had atrophy of the left cerebral peduncle, and functional studies showed his language lateralized to the right. He was recommended for functional hemispherotomy; the large left-sided cyst was favorable to endoscopic intervention, as it allowed a larger working space without retraction of brain parenchyma.

\section{Case 2}

A 23-month-old boy had a history of perinatal stroke and medically refractory epilepsy and encephalopathy, leading to developmental delay and failure to thrive. Comprehensive evaluation by the epilepsy team included electroencephalography studies showing frequent high-amplitude multifocal epileptiform activity over the entire right hemisphere consistent with right hemispheric epilepsy. Magnetic resonance imaging showed cystic encephalomalacia of the right middle cerebral artery territory (Fig. 7C and D). He had atrophy of the right cerebral peduncle, and functional studies confirmed his language lateralized to the left. The large cyst made the patient suitable for minimally invasive endoscopic functional hemispherotomy.

\section{Discussion}

We demonstrated the anatomical feasibility of complete hemispheric disconnection via a minimally invasive
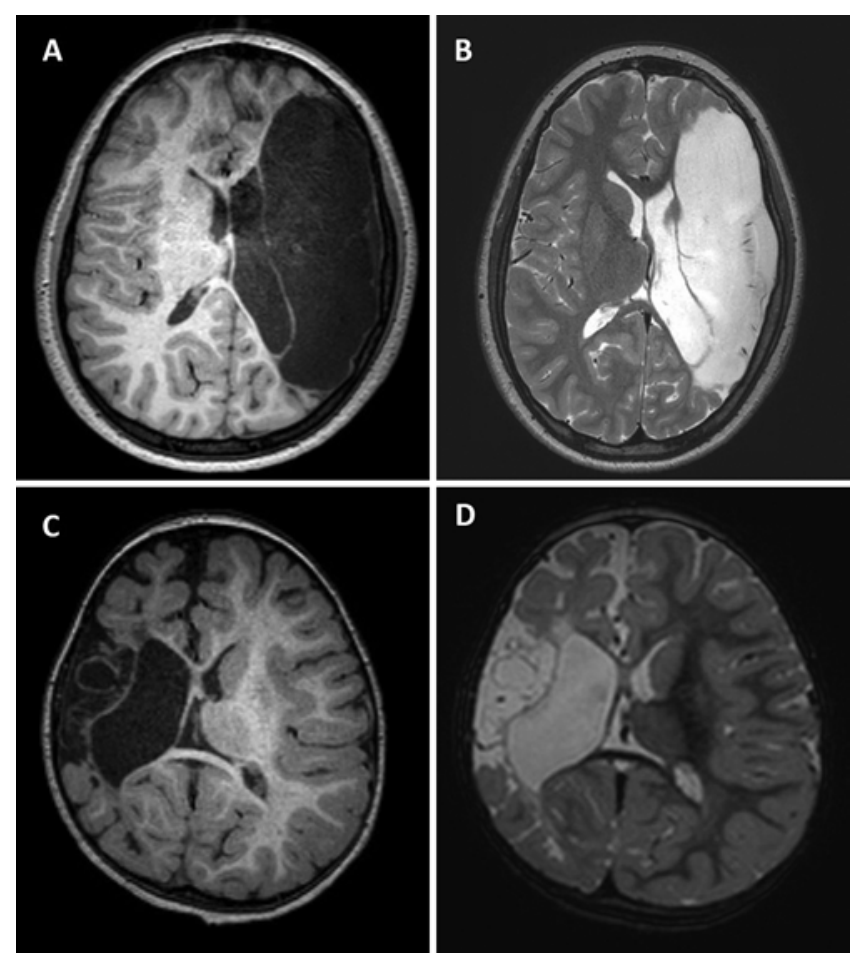

FIG. 7. Case 1. Preoperative axial T1-weighted (A) and T2-weighted (B) MR images. Case 2. Preoperative axial T1-weighted (C) and T2weighted (D) MR images.

endoscope-assisted technique in a cadaver study and subsequently translated this technique into clinical practice with encouraging preliminary results in carefully selected pediatric patients with intractable epilepsy.

Hemispherotomy has been a good surgical option in the treatment of medically refractory nonlesional epilepsy with seizures originating from one hemisphere. Classic techniques have required relatively extensive surgical exposure, which subjects children to the risks of bleeding, blood transfusion, fevers, postoperative aseptic meningitic symptoms, and prolonged recovery requiring extended hospitalization and inpatient rehabilitation stays. The advances of endoscopic and minimally invasive tools provide the opportunity for surgical techniques to also evolve in order to decrease perioperative morbidity and improve patient outcomes.

The anatomy of hemispherotomy has been well described. $7,13,18,24,25,31$ A thorough understanding of and comfort with this anatomy is essential, as the endoscopic approach uses trajectories and views structures from perspectives different from those previously described; thus, anatomical views may be unfamiliar and disorienting. However, we demonstrated that it is possible to clearly see and disconnect all critical structures using our cadaveric model. In that model, we also verified feasibility by developing back-up approaches to safely access required anatomical structures through additional burr holes and finally through standard craniotomy as necessary; these strategies were not needed in our two patients.

The callosotomy is the relatively straightforward component of the disconnection, as the working corridor is 
straight down along the falx, as in a standard interhemispheric approach to ventricular pathology. ${ }^{27}$ The anatomy is easily identified at the first ventricular entry into the ipsilateral ventricle, especially as most pediatric neurosurgeons are familiar with intraventricular neuroendoscopy. As the disconnection continues, maintaining orientation and identifying anatomical landmarks becomes imperative, as neuronavigation may lose accuracy with shifting of the brain tissue. A long rectangular cottonoid in the temporal horn serves as an indicator of this anatomical landmark, especially as the anatomy becomes distorted once CSF has been drained and the thin cortex of the affected hemisphere may be sagging from disconnection.

Our experience in the cadaver models allowed optimization of the technique and instrument manipulation in a bloodless field. As subpial resection via microsurgical technique requires clear visualization of specific fields with a rigid endoscope (genu of corpus callosum anteriorly, splenium posteriorly, temporal horn of the lateral ventricle lateral and deep), a dynamic working relationship between surgeon and operative assistant is essential. For instance, certain portions of the surgery require the endoscope angled from the anterior margin of the craniotomy with the surgeon working from the posterior portion of the opening. Conversely, the frontobasal disconnection was most easily performed with the endoscope entering from the posterior edge of the craniotomy and aimed anteriorly, with the surgeon's instruments working above the endoscope along the anterior edge of the craniotomy. All portions of the operative field were easily reached with our 4-cm craniotomy.

Several modifications were made in the transition to clinical application. Optimal positioning is essential for visualization of critical structures with the rigid endoscope. The head should be sufficiently flexed to allow for the appropriate angles required to visualize the anterior and posterior disconnection, but with vigilant assessment of the airway in conjunction with the anesthesia team to avoid obstruction. It is essential to plan for and prepare the field for a possible full craniotomy in the event that endoscopic access is suboptimal and if the goals of the surgery cannot be achieved safely. Neuronavigation is used, but surgeons should always be cognizant that technology can be unreliable, especially as anatomy shifts during surgery. Intraoperative ultrasound can theoretically be a useful adjunct in identifying known landmarks. However, no surgical navigation technology can substitute for experienced, intimate knowledge of the surgical anatomy. An angled camera on the endoscope is useful for simplifying the limited working space for the traffic of instruments and hands in the surgical corridor.

Previous studies have shown that patients in whom hemispherectomy has failed are likely to have remaining hemispheric connections, particularly anteriorly, and can benefit from reoperation to complete the disconnection. ${ }^{11}$ This new technique may raise concerns that complete disconnection may be inadequately evaluated with the endoscopic view compared to the direct visualization provided by classic open procedures; therefore, we have high standards of close inspection and verification of every disconnection step. We also carefully reviewed the intraoperative endoscopic video of every surgery and verified disconnec- tions radiographically with postoperative tractography in our patients.

Blood transfusions were not required. Postoperatively, hemispherotomy patients have external ventricular drainage to allow egress of any postoperative intraventricular blood and protein in the CSF. In our two cases, the drains were weaned and removed without incident, with rapidly decreasing output and low intracranial pressures. Patients recovered quickly, without the postoperative frontotemporal scalp flap soft-tissue swelling and pain that typically accompany open craniotomy. The paramedian incisions obviated temporalis muscle disruption. Both patients were discharged home without new neurological deficits and without the need for inpatient rehabilitation.

Recognizing limitations of the novel approach and equipment, as well as strengths and limitations of the operative team, is a crucial area of insight and understanding. For instance, patients with Sturge-Weber syndrome and hemimegalencephaly would not have been appropriate for initial deployment of this endoscope-assisted technology given our understanding that these cases are challenging even in standard open craniotomy approaches. Our first patients for this relatively novel technique had generously sized ventricles and thin cortical mantles. This allowed for a larger operative corridor and more working room than would be possible in the aforementioned pathologies. A learning curve is expected in the development of any new technique, and we expect to apply our technique to increasingly varied patients as workflow and surgical team skill improve.

Proper patient selection with thorough workup by a multidisciplinary epilepsy team is crucial in any epilepsy surgery. Careful patient selection is also essential in translating innovation in surgical technique into clinical practice. Establishing contingency plans to ensure the achievement of surgical goals, as well as head off any possible complications, is imperative. In surgery, the patients were positioned and marked for extending to standard open craniotomy, with defined intraoperative checkpoints for anatomy visualization and strict goals for complete disconnection. The surgical and anesthesia teams were prepared for possible complications as the surgeons worked around the venous sinuses and cranial vessels. Recognizing possible limitations and complications while applying rigor and safety to the development of this endoscopic surgical technique with subsequent translation into clinical care has been paramount to this process.

\section{Conclusions}

Full hemispheric disconnection can be completed with endoscopic assistance through a mini craniotomy, minimizing operative exposure. Magnetic resonance imaging with diffusion tensor imaging allows for verification of fiber tract disconnection. Further studies and long-term follow-up are needed to determine seizure freedom and complication rates compared to those in standard open craniotomy.

\section{References}

1. Bahuleyan B, Vogel TW, Robinson S, Cohen AR: Endoscopic 
total corpus callosotomy: cadaveric demonstration of a new approach. Pediatr Neurosurg 47:455-460, 2011

2. Bendjilali N, Nelson J, Weinsheimer S, Sidney S, Zaroff JG, Hetts SW, et al: Common variants on 9p21.3 are associated with brain arteriovenous malformations with accompanying arterial aneurysms. J Neurol Neurosurg Psychiatry 85:1280-1283, 2014

3. Binder DK, Schramm J: Transsylvian functional hemispherectomy. Childs Nerv Syst 22:960-966, 2006

4. Chandra SP, Kurwale NS, Chibber SS, Banerji J, Dwivedi $\mathrm{R}$, Garg A, et al: Endoscopic-assisted (through a mini craniotomy) corpus callosotomy combined with anterior, hippocampal, and posterior commissurotomy in Lennox-Gastaut syndrome: a pilot study to establish its safety and efficacy. Neurosurgery 78:743-751, 2016

5. Chandra SP, Tripathi M: Endoscopic epilepsy surgery: emergence of a new procedure. Neurol India 63:571-582, 2015

6. Dandy WE: Removal of right cerebral hemisphere for certain tumours with hemiplegia. JAMA 90:832-825, 1928

7. Delalande O, Bulteau C, Dellatolas G, Fohlen M, Jalin C, Buret V, et al: Vertical parasagittal hemispherotomy: surgical procedures and clinical long-term outcomes in a population of 83 children. Neurosurgery 60 (2 Suppl 1):ONS19ONS32, 2007

8. Devlin AM, Cross JH, Harkness W, Chong WK, Harding B, Vargha-Khadem F, et al: Clinical outcomes of hemispherectomy for epilepsy in childhood and adolescence. Brain 126:556-566, 2003

9. Falconer MA, Wilson PJ: Complications related to delayed hemorrhage after hemispherectomy. J Neurosurg 30:413426,1969

10. Griessenauer CJ, Salam S, Hendrix P, Patel DM, Tubbs RS, Blount JP, et al: Hemispherectomy for treatment of refractory epilepsy in the pediatric age group: a systematic review. J Neurosurg Pediatr 15:34-44, 2015

11. Kiehna EN, Widjaja E, Holowka S, Carter Snead O III, Drake J, Weiss SK, et al: Utility of diffusion tensor imaging studies linked to neuronavigation and other modalities in repeat hemispherotomy for intractable epilepsy. J Neurosurg Pediatr 17:483-490, 2016

12. Krynauw RA: Infantile hemiplegia treated by removing one cerebral hemisphere. J Neurol Neurosurg Psychiatry 13:243-267, 1950

13. Kucukyuruk B, Yagmurlu K, Tanriover N, Uzan M, Rhoton AL Jr: Microsurgical anatomy of the white matter tracts in hemispherotomy. Neurosurgery 10 (Suppl 2):305-324, 2014

14. Lew SM, Koop JI, Mueller WM, Matthews AE, Mallonee JC: Fifty consecutive hemispherectomies: outcomes, evolution of technique, complications, and lessons learned. Neurosurgery 74:182-195, 2014

15. Lin Y, Harris DA, Curry DJ, Lam S: Trends in outcomes, complications, and hospitalization costs for hemispherectomy in the United States for the years 2000-2009. Epilepsia 56:139-146, 2015

16. Marras CE, Granata T, Franzini A, Freri E, Villani F, Casazza M, et al: Hemispherotomy and functional hemispherectomy: indications and outcome. Epilepsy Res 89:104-112, 2010

17. McKenzie K: The present status of a patient who had the right hemisphere removed. JAMA 111:168, 1938

18. Morino M, Shimizu H, Ohata K, Tanaka K, Hara M: Anatomical analysis of different hemispherotomy procedures based on dissection of cadaveric brains. J Neurosurg 97:423-431, 2002
19. Nasi D, Iacoangeli M, Di Somma L, Dobran M, Di Rienzo A, Gladi M, et al: Microsurgical endoscopy-assisted anterior corpus callosotomy for drug-resistant epilepsy in an adult unresponsive to vagus nerve stimulation. Epilepsy Behav Case Rep 5:27-30, 2016

20. Oppenheimer DR, Griffith HB: Persistent intracranial bleeding as a complication of hemispherectomy. J Neurol Neurosurg Psychiatry 29:229-240, 1966

21. Ramantani G, Kadish NE, Brandt A, Strobl K, Stathi A, Wiegand $\mathrm{G}$, et al: Seizure control and developmental trajectories after hemispherotomy for refractory epilepsy in childhood and adolescence. Epilepsia 54:1046-1055, 2013

22. Rasmussen T: Hemispherectomy for seizures revisited. Can J Neurol Sci 10:71-78, 1983

23. Rasmussen T: Postoperative superficial hemosiderosis of the brain, its diagnosis, treatment and prevention. Trans Am Neurol Assoc 98:133-137, 1973

24. Schramm J, Behrens E: Functional hemispherectomy. J Neurosurg 87:801-802, 1997

25. Schramm J, Kral T, Clusmann H: Transsylvian keyhole functional hemispherectomy. Neurosurgery 49:891-901, 2001

26. Shimizu H, Maehara T: Modification of peri-insular hemispherotomy and surgical results. Neurosurgery 47:367-373, 2000

27. Smyth MD, Vellimana AK, Asano E, Sood S: Corpus callosotomy-Open and endoscopic surgical techniques. Epilepsia 58 (Suppl 1):73-79, 2017

28. Sood S, Marupudi NI, Asano E, Haridas A, Ham SD: Endoscopic corpus callosotomy and hemispherotomy. J Neurosurg Pediatr 16:681-686, 2015

29. Tanriverdi T, Olivier A, Poulin N, Andermann F, Dubeau F: Long-term seizure outcome after corpus callosotomy: a retrospective analysis of 95 patients. J Neurosurg 110:332-342, 2009

30. van der Kolk NM, Boshuisen K, van Empelen R, Koudijs SM, Staudt M, van Rijen PC, et al: Etiology-specific differences in motor function after hemispherectomy. Epilepsy Res 103:221-230, 2013

31. Villemure J, Rasmussen T: Functional hemispherectomy: methodology. J Epilepsy 3 Suppl:177-182, 1990

32. Villemure JG, Mascott CR: Peri-insular hemispherotomy: surgical principles and anatomy. Neurosurgery 37:975-981, 1995

\section{Disclosures}

The authors report no conflict of interest concerning the materials or methods used in this study or the findings specified in this paper.

\section{Author Contributions}

Conception and design: Lam. Acquisition of data: Lam, Wagner, Vaz-Guimaraes. Analysis and interpretation of data: Lam, Wagner. Drafting the article: Lam, Wagner. Critically revising the article: Lam, Wagner. Reviewed submitted version of manuscript: Lam, Wagner. Approved the final version of the manuscript on behalf of all authors: Lam. Administrative/technical/material support: Lam, Vaz-Guimaraes, Camstra. Study supervision: Lam.

\section{Correspondence}

Sandi Lam: Baylor College of Medicine, Texas Children's Hospital, Houston TX. sklam@texaschildrens.org. 